\title{
A gift for unraveling complexity
}

\section{London}

Although Pierre-Gilles de Gennes of the College de France won this year's Nobel prize in physics for his work on polymers and liquid crystals, the decision reflects just as much his contribution throughout condensed matter physics. His work has been instrumental in defining the topic

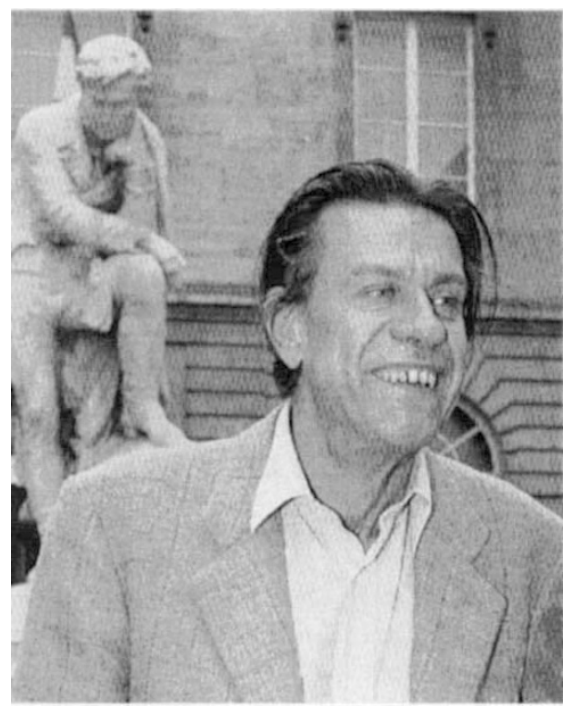

Father of the LCD display itself - that is, in demonstrating that principles can be identified which describe the behaviour of condensed systems as diverse as magnets, superconductors and assemblies of macromolecules.

De Gennes is known for his ability to see the underlying physics in systems of seemingly intractable complexity. In this sense, his work follows the spirit of that of the Soviet physicist Lev Landau, who showed that phase transitions in disparate media commonly share universal features.

De Gennes' early work on phase transitions involved the "classical" problem of ordering phenomena in sold-state magnets, and he contributed further to the understanding of "hard" condensed systems with work on superconductivity. But it is his research into soft condensed phases that has had the greatest impact, particularly those - such as polymers and liquid crystals - for which the generic term "complex fluids" has now become fashionable. De Gennes' most telling insight lay in the proposal that concepts developed to explain classical phase transitions could be carried over to these considerably less tidy systems.

Working at the University of Orsay, de Gennes showed that transitions between ordered phases of liquid crystals bore analogies to the transition from a normal to a superconducting state. In transforming from a so-called cholesteric to a smectic phase, liquid-crystal molecules initially aligned in a twisted manner lose this twist and become ordered into a parallel array.

Transitions in liquid crystals have at least the simplifying characteristic that the molecules tend to align collectively into ordered structures. Polymers rarely show such cooperatively but instead display a disordered tangle of chains. Yet de Gennes succeeded in extending to polymer solutions the ideas developed to describe phase transitions in much "cleaner" systems. $\mathrm{He}$ showed that the physical properties of polymers obey "scaling laws." Scaling is a central tenet of the modern theory of phase transitions.

In surface science, de Gennes advanced the understanding of wetting dynamics by providing a theory for how liquid droplets spread on smooth and rough substrates. His work on the adsorption of polymers at solid surfaces has now led him to display an interest in applied fields such as tribology and adhesion - traditionally engineering disciplines, to which de Gennes will no doubt show that condensed matter physics has a contribution to make.

Philip Ball

\section{CHEMISTRY \\ Bringing NMR to the masses}

\section{London}

Completing a clean sweep by European researchers of this year's science Nobel Prizes, the 1991 award for chemistry has gone to Richard Ernst of the Swiss Federal Institute of Technology in Zürich. Ernst is responsible for two refinements that helped to transform nuclear magnetic resonance spectroscopy (NMR) from a specialist tool into a staple of modern chemistry.

Before Ernst's work, NMR involved applying a slow sweep of varying radio frequencies to a chemical sample held in a magnetic field. At particular frequencies, the spins of many atomic nuclei reorientate themselves with respect to the magnetic field, which induces an electric signal in the detectors of an NMR spectrometer. Because these 'resonance frequencies' vary according to the chemical environment in which the nuclei find themselves, the pioneers of the technique realized that NMR could be used in chemical analysis. Early NMR spectrometers, however, lacked sensitivity. Only a handful of nuclei - principally hydrogen, fluorine and phosphorus - yielded signals strong enough to distinguish their resonance frequencies reliably.

In the mid-1960s, Ernst dramatically increased the sensitivity of NMR by replacing the gentle sweep of varying fre- quencies with short, intense radio pulses. Ken Packer, from British Petroleum's Sunbury Research Centre, likens the approach to "hitting a piano with a hammer", rather than pressing each key in sequence. By applying a mathematical operation called a Fourier transformation to the complex signal given off by the nuclei, Emst was able to reconstruct standard NMR spectra.

The Fourier transformation method increased NMR's sensitivity by more than tenfold, greatly expanding the number of different nuclei that could be studied. Most important, chemists could for the first time reliably determine the resonance frequency of carbon-13 nuclei, which yield useful NMR spectra (unlike the more common carbon-12) but which were present in insufficient quantities in chemical samples to be analysed successfully using the first NMR spectrometers.

Ernst's second major contribution was to pioneer two-dimensional NMR. Instead of using single radio-frequency pulses, Ernst and his colleagues began to experiment with sequences of pulses. In twodimensional NMR, samples are repeatedly subjected to a pair of pulses in close succession, and the interval between the pulses in each pair is varied. The resulting signals can then be measured both as a function of time during each detection (as

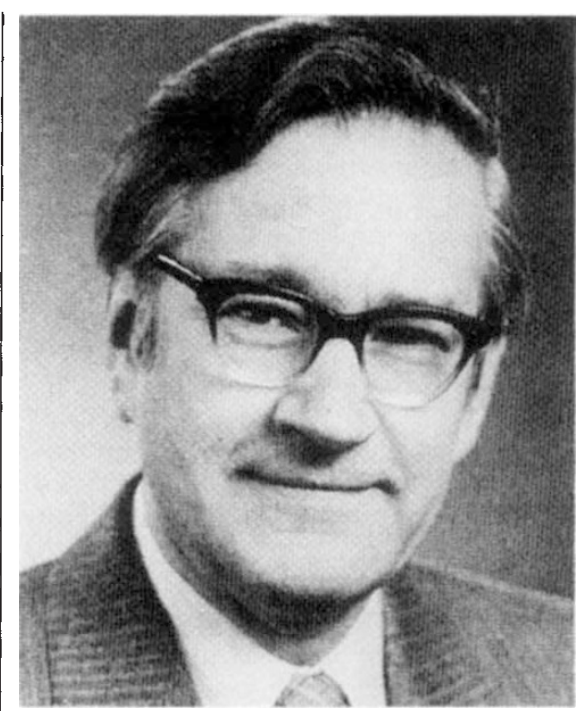

Explored the mathematics of resonance

in Fourier transform NMR), and as a function of the interval between the two pulses in each pair. These data then give twodimensional spectra that can show which nuclei in a molecule lie close together.

This new development allowed NMR to be applied to the study of larger molecules than was previously possible. The result was that NMR became a key tool in biochemistry, launching a new range of structural studies of biological molecules in solution, and even of their interactions with drugs and metal ions. Peter Aldhous 